\title{
Dandy-Walker Malformation: Prenatal sonographic diagnosis
}

\section{Konstantinos Zacharis, Stavros Kravvaritis, Anastasia Papakonstantinou, Theodoros Charitos, Eleni Chrysafopoulou, Anastasia Fouka}

Department of Obstetrics and Gynecology, General Hospital of Lamia, Lamia, Greece

\section{Corresponding Author}

General Hospital of Lamia, Papasiopoulou (end), Lamia, 35131, Greece; tel:+302231356189; email: zaxarisk@yahoo.com

\section{Abstract}

The Dandy-Walker malformation is a rare congenital intracranial malformation that comprises of complete or partial agenesis of the cerebellar vermis, cystic dilatation of the 4 th ventricle and an enlarged posterior fossa. Here we report a case of Dandy-Walker malformation detected incidentally during a routine fetal sonographic examination at 18 weeks of gestation. The couple decided termination of pregnancy, after adequate genetic counseling. Detailed postmortem examination revealed correlation between prenatal sonographic imaging and histopathological findings. This case report indicates the fact that fetal magnetic resonance imaging may not be necessary in diagnosis, especially when pregnancy termination is preferred.

Key words: Dandy-walker malformation, posterior fossa anomaly, prenatal diagnosis, ultrasound

\section{Introduction}

Dandy-Walker malformation (DWM) is a posterior fossa anomaly described as vermian hypoplasia or agenesis and cystic dilatation of the $4^{\text {th }}$ ventricle causing upward displacement of tentorium and torcula ${ }^{1}$. Prenatal diagnosis of DWM is easily established by ultrasound and/or magnetic resonance imaging (MRI). Although, thorough evaluation of the fetus with DWM is mandatory in order to reach a final diagnosis, evaluate prognosis, and give appropriate genetic counseling ${ }^{2}$. We hereby report a case of DWM diagnosis during routine antenatal ultrasonography at 18 weeks of gestation and pregnancy's outcome.

\section{Case report}

A 21-year-old primigravida woman attended our outpatient department for routine antenatal ultrasound at 18 weeks of gestation. The patient had no symptoms to offer and abdominal examination revealed uterine size of 18-19 weeks.

We were informed that patient did not undergo nuchal translucency scan during the first trimester. She performed the triple screen during 15 weeks of gestation. Results indicated a high risk for Trisomy 13 and $18(1 / 58$ with negative limit: $<1 / 100)$, hence amniocentesis was suggested but the couple decided not to undergo. 
Ultrasound examination showed a viable fetus with weight at $29^{\text {th }}$ percentile for gestational age. Ultrasound also revealed vermian agenesis, cystic dilatation of the fourth ventricle, enlargement of the posterior fossa but no evidence of hydrocephalus (Figure 1). These ultrasound findings demonstrated DWM. There was no sonographic evidence of any other extra-CNS congenital malformation. For further evaluation, fetal MRI was recommended but the couple did not wish to undergo.

There was no family history of genetic or neurological disorders. Maternal screening for toxoplasmosis, rubella, cytomegalovirus, herpes simplex virus (TORCH) and diabetes mellitus testings were negative.

Woman along with her husband were referred for genetic counseling. The parents opted for pregnancy termination and gave consent for autopsy. The patient was administered $400 \mathrm{mcg}$ of misoprostol intravaginal every 3 hours. After 8 hours a medical

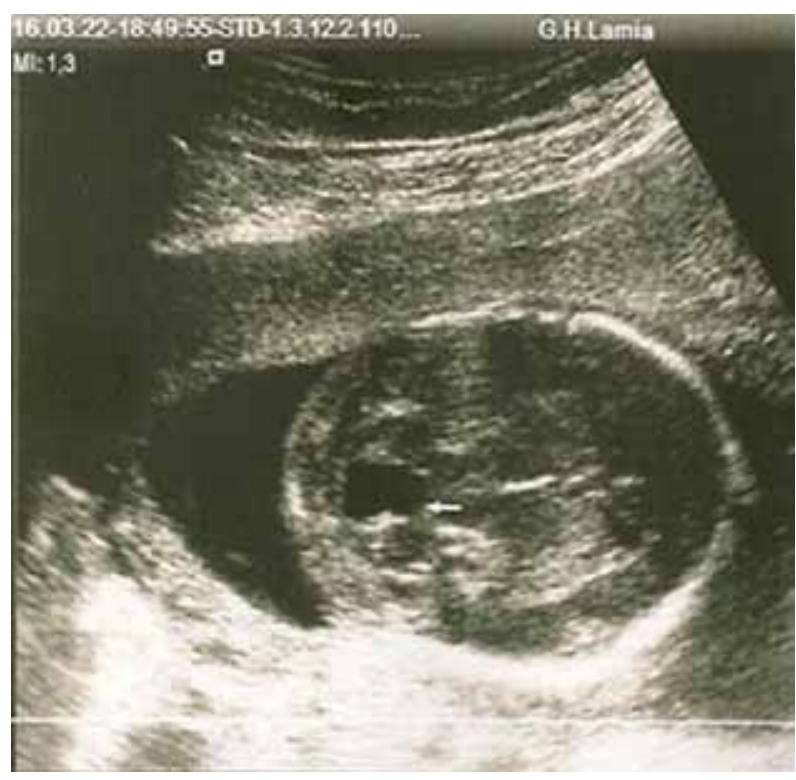

Figure 1. Axial sonogram shows the enlarged posterior fossa, small cerebellum with splayed cerebellar hemispheres and agenesis of the vermis. induced miscarriage occurred, followed by curettage.

Detailed postmortem examination was carried out, however genetic testing was not performed. The male fetus weighed $340 \mathrm{~g}$ with a crown to rump length of $18 \mathrm{~cm}$, which were consistent with a gestational age of 20 weeks. Internal examination did not show any other abnormality. Neuropathological examination demonstrated a large cyst, which occupied the posterior fossa, and the cerebellar hemispheres were widely separated with complete agenesis of the vermis. Placental examination revealed extensive calcium deposits and a three-vessel umbilical cord was also reported. Hence, a diagnosis of DWM was confirmed.

\section{Discussion}

The DWM is a term which represents several abnormalities of brain development which coexist and not just a single entity. This malformation is a congenital brain disorder commonly involving the cerebellum and the fourth ventricle ${ }^{3}$. In the United States, DWM and related variants have a prevalence of 1 in 35000 live births and they account for approximately 1 to $4 \%$ of hydrocephalus cases ${ }^{1}$. Mortality rate is estimated between 12 and $50 \%$ and fetal mortality is correlated with the presence of extraCNS anomalies ${ }^{4,5}$. Although the majority of cases are sporadic, some may result from chromosomal aberrations, Mendelian disorders, fetal alcohol exposure, congenital rubella and maternal diabetes ${ }^{6,7}$.

Prenatal diagnosis of DWM by ultrasonography is easily achieved as it is recognized by an enlarged posterior fossa, cystic mass of the posterior fossa communicating with the fourth ventricle and varying degree of hypoplasia/agenesis of the vermis ${ }^{3}$. It is also essential to differentiate from other posterior fossa anomalies like retrocerebellar arachnoid cysts, Blake's pouch cyst, mega cisterna magna, hence an MRI may be necessary ${ }^{8,9}$. In our case, all these anomalies were observed on ultrasound examination 
and therefore there was no need to perform MRI in order to confirm the DWM.

Diagnosis and prognosis may be difficult to assess, provided that DWM comprises a challenging clinical entity. In patients with DWM characterized by partial vermian agenesis and absence of associated anatomical anomalies, a potential good clinical and intellectual outcome has been demonstrated in recent literature ${ }^{10}$. However, termination of pregnancy remains couple's decision, once adequate counseling has been given.

In conclusion, this case report documents a DWM in a 20-week male fetus with correlation between prenatal sonographic imaging and histopathological findings. It also highlights that ultrasonography can be used as the only method for close monitoring antenatally, since MRI was not performed. Finally, neuropathological examination is required in order to confirm this syndromic entity.

\section{Consent}

Informed consent was obtained

\section{Conflicts}

The authors declare no conflicts of interest

\section{Author's contribution}

$\mathrm{KZ}$, AP and EC wrote the first draft. SK, TC and AF critically reviewed and amended the draft. All authors approved of the final draft

\section{References}

1. Zamora EA, Ahmad T. Dandy Walker Malformation. [Updated 2019 Nov 17]. In: StatPearls [Internet]. Treasure Island (FL): StatPearls Publishing; 2020 Jan-. Available from: https://www.ncbi. nlm.nih.gov/books/NBK538197/.

2. Darouich S, Amraoui J, Amraoui N. Prenatal sonographic diagnosis of Dandy-Walker malformation and type III lissencephaly: A novel associa- tion. J Clin Ultrasound. 2019 Dec 20. doi: 10.1002/ jcu.22805.

3. Alam A, Chander BN, Bhatia M. Dandy-Walker Variant : Prenatal Diagnosis by Ultrasonography. Med J Armed Forces India. 2004;60(3):287-289. doi:10.1016/S0377-1237(04)80066-2.

4. Treviño Alanís MG, González Cantú N, Montes Cruz JV, García Flores JB, Martínez Menchaca HR, Rivera Silva G. [Dandy Walker malformation]. Arch Argent Pediatr. 2014 Feb;112(1):103-4. doi: 10.1590/ S0325-00752014000100024.

5. Nyberg DA, Cyr DR, Mack LA, Fitzsimmons J, Hickok D, Mahony BS. The Dandy-Walker malformation prenatal sonographic diagnosis and its clinical significance. J Ultrasound Med. 1988 Feb;7(2):65-71.

6. Stambolliu E, Ioakeim-Ioannidou M, Kontokostas K, Dakoutrou M, Kousoulis AA. The Most Common Comorbidities in Dandy-Walker Syndrome Patients: A Systematic Review of Case Reports. J. Child Neurol. 2017 Sep;32(10):886-902. doi: 10.1177/0883073817712589. Epub 2017 Jun 21.

7. Spennato P, Mirone G, Nastro A, Buonocore MC, Ruggiero C, Trischitta $V$, et al. Hydrocephalus in Dandy-Walker malformation. Childs Nerv Syst. 2011;27:1665. doi: 10.1007/s00381-011-15444. Epub 2011 Sep 17.

8. D’Antonio F, Khalil A, Garel C, Pilu G, Rizzo G, Lerman-Sagie T, et al. Systematic review and meta-analysis of isolated posterior fossa malformations on prenatal ultrasound imaging (part 1): nomenclature, diagnostic accuracy and associated anomalies. Ultrasound Obstet Gynecol. 2016 Jun;47(6):690-7. doi: 10.1002/uog.14900. Epub 2016 May 1.

9. Wüest A, Surbek D, Wiest R, et al. Enlarged posterior fossa on prenatal imaging: differential diagnosis, associated anomalies and postnatal outcome. Acta Obstet Gynecol Scand. 2017;96:837. doi: 10.1111/aogs.13131. Epub 2017 Apr 10.

10. Guibaud L, Larroque A, Ville D, Sanlaville D, Till 
M, Gaucherand P, et al. Prenatal diagnosis of 'isolated' Dandy-Walker malformation: imaging findings and prenatal counselling. Prenat Diagn. 2012 Received 27-03-20

Feb;32(2):185-93. doi: 10.1002/pd.3828.

Revised 07-05-20

Accepted 07-05-20 